\title{
Fluoroscopically guided transforaminal epidural dry needling for lumbar spinal stenosis using a specially designed needle
}

\author{
Kang Ahn' ${ }^{1}$, Hyung-Joon Jhun ${ }^{2 *}$, Tae-Kyun Lim¹, Yong-Seung Lee ${ }^{1}$
}

\begin{abstract}
Background: This report describes the methodological approach and clinical application of a minimally invasive intervention to treat lumbar spinal stenosis (LSS).

Methods: Thirty-four patients with LSS underwent fluoroscopically guided transforaminal epidural dry needling using a specially designed flexed Round Needle. The needle was inserted $8-12 \mathrm{~cm}$ lateral to the midline at the level of the stenosis and advanced to a position between the anterior side of the facet joint and pedicle up to the outer-third of the pedicle. The needle was advanced medially and backed laterally within a few millimetres along the canal side of the inferior articular process between the facet joint and pedicle. The procedure was completed when a marked reduction in resistance was felt at the tip of the needle. The procedure was performed bilaterally at the level of the stenosis.

Results: The average follow-up period was $12.9 \pm 1.1$ months. The visual analogue scale (VAS) pain score was reduced from $7.3 \pm 2.0$ to $4.6 \pm 2.5$ points, the Oswestry Disability Index (ODI) score decreased from $41.4 \pm 17.2$ to $25.5 \pm 12.6 \%$ and the average self-rated improvement was $52.6 \pm 33.1 \%$. The VAS scores indicated that 14 (41.2\%) patients reported a "good" to "excellent" treatment response, while 11 (32.4\%) had a "good" to "excellent" treatment response on the ODI and 22 (64.7\%) had a "good" to "excellent" treatment response on the self-rated improvement scale.
\end{abstract}

Conclusions: These results suggest that fluoroscopically guided transforaminal epidural dry needling is effective for managing LSS.

\section{Background}

Lumbar spinal stenosis (LSS) is a painful and potentially disabling condition that is defined as a narrowing of the lumbar spinal canal, nerve root canal or intervertebral foramina. It is often encountered in the geriatric population. The primary causes of spinal canal constriction are a protruding intervertebral disc, hypertrophied facet joint and thickened ligamentum flavum [1].

Patients suffering from LSS develop pain, paraesthesias, numbness and weakness in the back and legs caused by compression of the lumbosacral nerve roots in the constricted neural canal and foramina [2]. Neurogenic claudication, the most common symptom of LSS,

\footnotetext{
* Correspondence: oemdoc21@gmail.com

${ }^{2}$ Centre for Interventional Pain Management, Daejeon Woori Hospital, 1419,

Metrozone, Doonsan-Dong, Seo-Ku, Daejeon, 302-531, Republic of Korea

Full list of author information is available at the end of the article
}

is the progressive onset of radicular pain, paraesthesias and numbness and may cause weakness during walking or be worsened by walking. LSS is exacerbated by lumbar extension and improves with lumbar flexion [3].

A wide variety of conservative methods has been developed to treat LSS. They include medication, physical therapy, behavioural therapy, orthopaedic devices, girdles, acupuncture and manual therapy. Patients refractory to conservative therapy generally undergo surgery to decompress the spinal canal and the neural foramina, and eliminate pressure on the spinal nerve roots $[4,5]$.

We developed a minimally invasive interventional technique to treat LSS. The present study illustrates the methodological approach and clinical application of this technique. 


\section{Methods \\ Subjects}

The subjects were 34 consecutive patients ( 9 men and 25 women) with LSS who underwent fluoroscopically guided transforaminal epidural dry needling at a chronic-pain management centre in Korea in 2008. Patients included in the study had received at least one treatment session and 12 or more months had elapsed since their last session.

The diagnosis of LSS was made based on clinical history, physical examination and three-dimensional computed tomography (3-D CT) findings. The inclusion criteria were back pain and/or radiating pain to the lower leg, accompanied by neurogenic claudication, exacerbated by lumbar extension and relieved by lumbar flexion; altered physical findings corresponding to chronic neuropathic pain and spinal stenosis, such as localised vasoconstriction, hair loss or trophoedema, limitation of extension and the presence of lumbar tenderness [6,7], and a reduction in the cross-sectional area of the spinal canal and neural foramina accompanied by degenerative changes of the lumbar spine, such as disk protrusion, hypertrophy of the ligamentum flavum, hypertrophy of the facet joints or the appearance of osteophytes on the 3-D CT. Studies have reported a high prevalence of abnormalities on imaging, including spinal stenosis in asymptomatic subjects and mismatching between symptoms and image findings $[8,9]$. Therefore, we evaluated whether the CT findings of individual lumbar spinal levels sufficiently corresponded to the patient's history and physical findings when determining treatment level(s). If a patient had symptoms corresponding to LSS and altered physical and abnormal CT findings were detected simultaneously for a specific lumbar joint, we deemed that level the treatment target of the intervention.

All of the patients were provided with comprehensive information on the benefits and potential risks (such as infection, bleeding, post-needling soreness and dural puncture) of the intervention and gave informed consent prior to the treatment. A follow-up evaluation was conducted at hospital 3 weeks after the treatment. If a patient complained of persistent pain or limited improvement, the treatment was repeated at 3-week intervals.

As this study was a follow-up of patients who had previously received the intervention, written informed consent was not required. The institutional review board approved the study protocol.

\section{Needle and procedure}

Many studies have demonstrated the anatomy of the lumbar spine [10] and the pathophysiological mechanisms of
LSS [11-13]. Based on these studies, we postulated that the posterior epidural space adjacent to the intervertebral foramen that lies between the anterior side of the facet joint and pedicle in the sagittal plane (Figure 1A), usually up to the outer-third of the pedicle in the coronal plane (Figure 1B) and between the ligamentum flavum and the dural sleeve in the axial plane (Figure $1 C$ ) should be the primary treatment target in LSS. However, additional treatment was performed in the anterior epidural space if the posterior epidural approach did not produce a favourable treatment outcome.

We treated LSS using a specially designed needle (Round Needle ${ }^{\oplus}$ Hansung Precision, Korea) that is 1.2 $\mathrm{mm}$ in diameter and $120 \mathrm{~mm}$ long (Figure 2A). The needle is streamlined, solid and flexible, and has a blunt, round tip [14] (Figures $2 \mathrm{~B}$ and $2 \mathrm{C}$ ). We bent the needle $5-30^{\circ}$ at a point $2-4 \mathrm{~mm}$ from the tip (Figures 2D, E and $2 \mathrm{~F}$ ) using surgical tweezers to allow it to accurately reach the target structures and avoid damage to the spine, particularly possible damage to arteries because of anatomical variation or neovascularisation of the lesion [15]. The degree of bending was determined after identifying the shape of the intervertebral foramen on the axial CT image.

The patients were instructed to lie on a table in the prone position. A C-arm fluoroscope was used to identify the bony landmark in the anteroposterior view and the skin was marked and cleaned. Local anaesthesia was achieved with $1 \%$ lidocaine; however, if patients experienced severe pain or became irritable during the procedure, they were sedated with intravenous midazolam or propofol.

At the level of the stenosis, the flexed Round Needle was inserted 8-12 cm lateral to the midline with the concave surface facing up. The needle was advanced at a $15-30^{\circ}$ angle to the horizontal plane until the flexed tip contacted the lumbar spine (Figure 3A). Then the $\mathrm{C}$-arm was turned to the lateral view. The tip of the needle was positioned between the anterior side of the facet joint and pedicle (Figure 3B), and was then advanced to the outer-third of the pedicle (Figure 1B). It was advanced further, to the inner line of pedicle, as an additional treatment for the anterior epidural space if the previous posterior epidural approach did not produce a favourable treatment outcome. The needle was advanced medially and backed laterally a few millimetres along the canal side of the inferior articular process between the anterior side of the facet joint and pedicle. The procedure was complete when we felt a marked reduction in resistance at the tip of the needle. The procedure was performed bilaterally at each level of the stenosis (Additional file 1: A movie demonstrating the technique). 


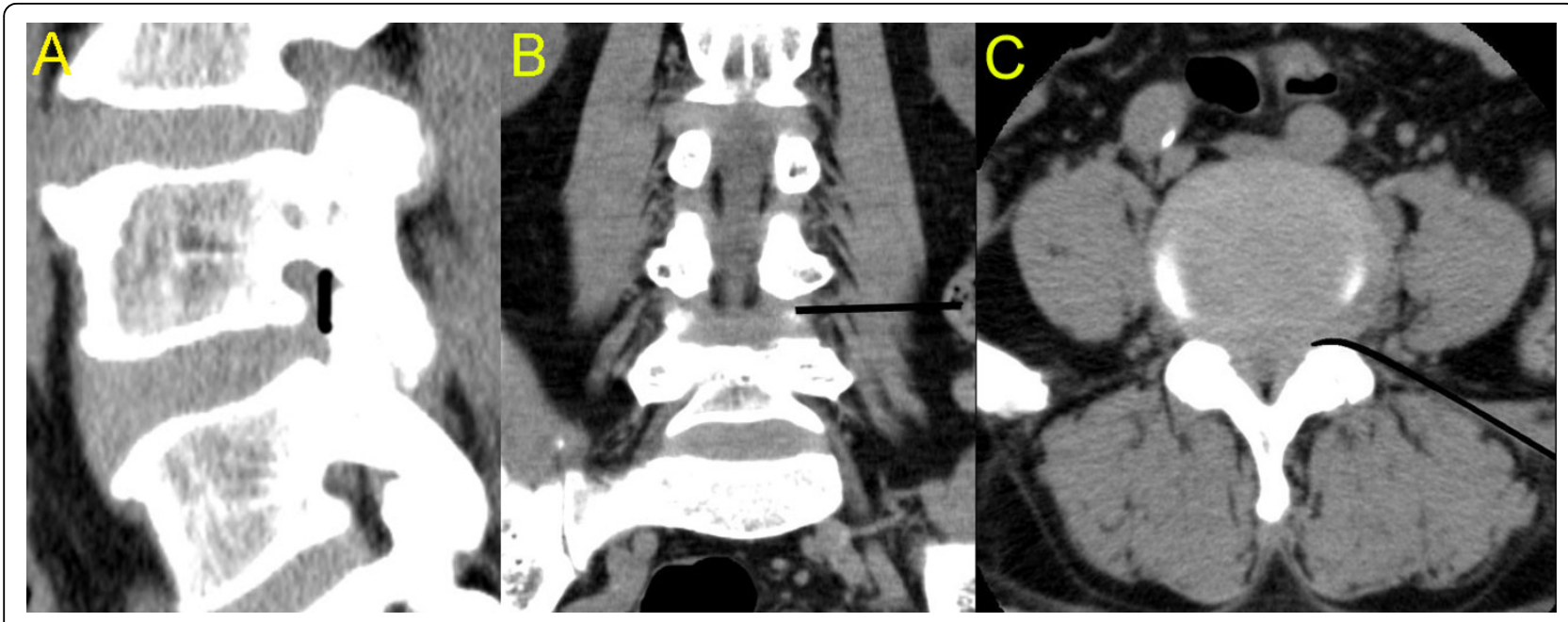

Figure 1 Three-dimensional CT images of the transforaminal epidural dry needling procedure. The sagittal CT image shows the area where the transforaminal epidural dry needling was performed (A). The coronal (B) and axial (C) CT images show the depth and route of needle insertion.

\section{Outcome measures}

We selected three outcome measures to evaluate the effectiveness of the treatment: a self-rated pain score, the Oswestry Disability Index (ODI) and self-rated improvement following the intervention. The self-rated pain score and the ODI were the primary outcomes and were assessed before and after treatment. Self-rated improvement was a secondary measure and assessed only after treatment.
The patients were asked to rate their pain level using a visual analogue scale (VAS) that consisted of a $10-\mathrm{cm}$ line anchored by two extremes. The ODI is one of the most common condition-specific outcome measures used for the management of spinal disorders [16]. It consists of 10 sections related to back pain and level of function: pain intensity, personal care, lifting, walking, sitting, standing, sleeping, sex life, social life and travelling. In each section, the patient is asked to choose the

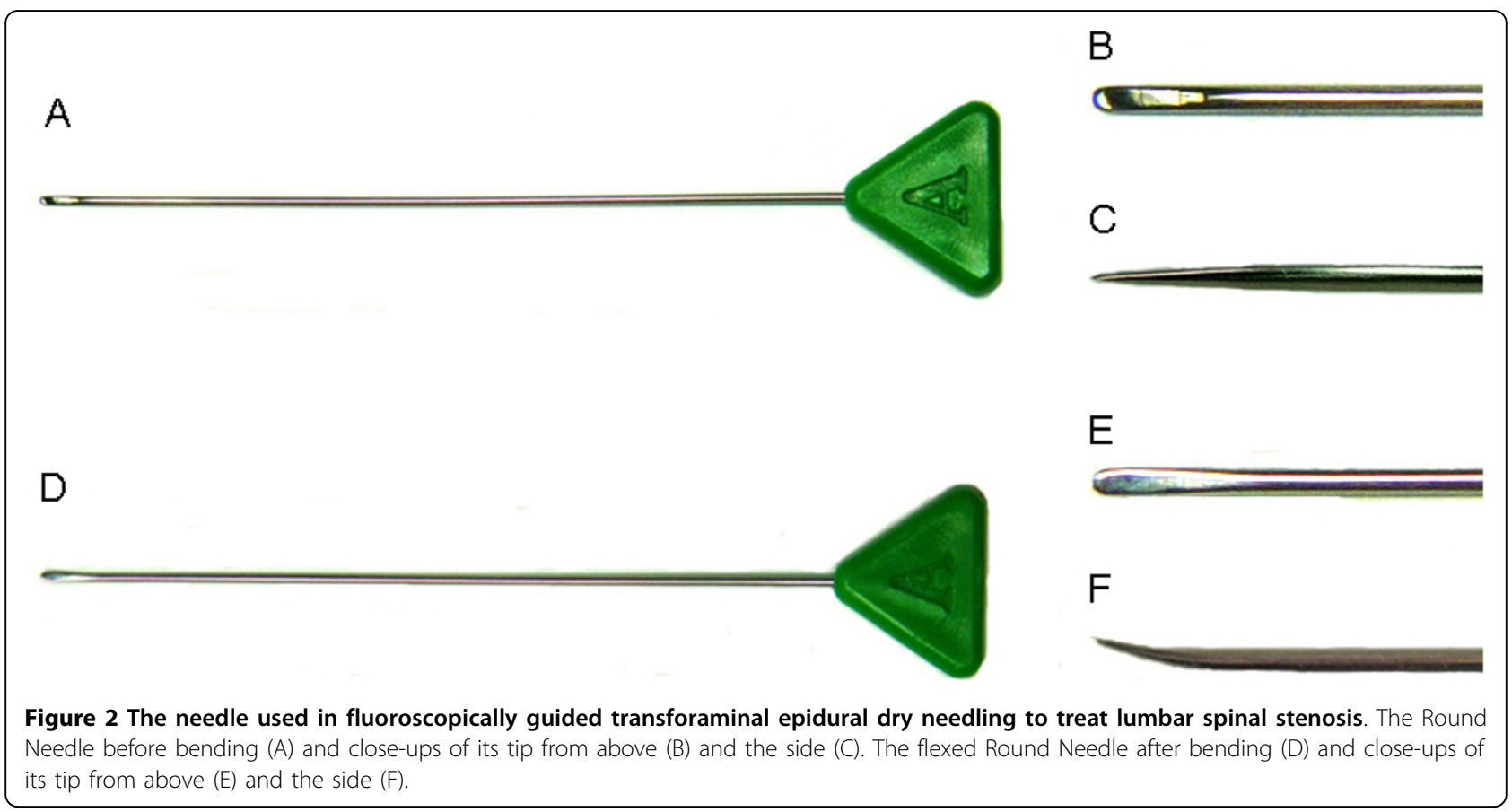




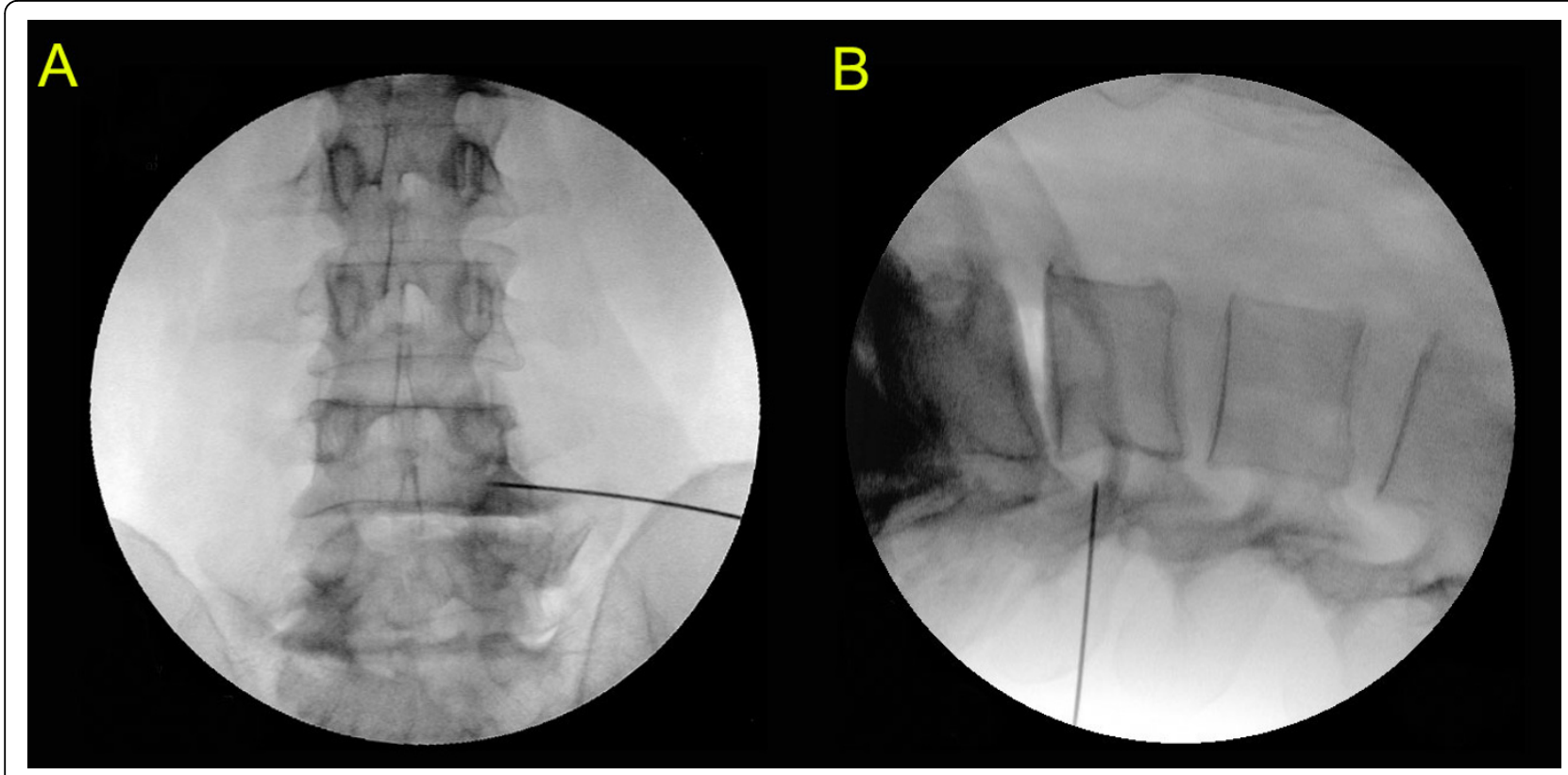

Figure 3 Fluoroscopy images of the transforaminal epidural dry needling procedure. The anteroposterior (A) and lateral (B) fluoroscopy images are from a patient undergoing the intervention.

statement that best describes his/her status. The statements are scored from 0 to 5 , corresponding to the level of limitation; 0 indicates the least limitation and 5 indicates the greatest limitation in each category. The total score can range from 0 (highest level of function) to 50 (lowest level of function). Some patients did not respond to every section; thus, the per cent disability was calculated on the basis of the total possible points. For example, if all 10 sections were completed, the total per cent disability was calculated as (total points/50) $\times 100$. If one section was missed or not applicable, the per cent disability was calculated as (total points/45) $\times 100$ [17]

The pre-interventional baseline data were obtained using a self-administered questionnaire when the patients initially visited hospital. The post-interventional follow-up data were obtained using a telephone survey. The follow-up outcome measures consisted of 12 items. It took 5-10 minutes to respond to all items. Medical research, including a follow-up study of musculoskeletal intervention, occasionally uses telephone surveys to obtain data, and arguments against the validity of such methods have not been raised [18]. To ensure the validity of the follow-up data, an independent researcher conducted the survey using a structured questionnaire in a standardised manner.

The Wilcoxon signed-rank test was used to evaluate the differences between pre- and post-intervention selfrated pain and per cent disability on the ODI scores. The null hypothesis of no improvement after treatment was tested to assess self-rated improvement following treatment. Additionally, we evaluated the per cent change of the individual outcome measures. The selfrated improvement score and the per cent change on the VAS and ODI scores were defined as follows: $\geq 75 \%$ was "excellent", a response of $50-74 \%$, was "good", $25-49 \%$, was "fair" and < 25\% was "poor".

\section{Results}

The average age of the subjects was $62.9 \pm 11.2$ years $(57.7 \pm 14.5$ years for the men, $64.8 \pm 9.3$ for the women). The average duration of pain prior to the treatment was $68.2 \pm 80.9$ months $(86.3 \pm 133.1$ months for the men, $61.7 \pm 54.0$ months for the women). The affected levels were L4-L5 in 31 (91.2\%), L3-L4 in 19 (55.9\%), L5-S1 in 15 (44.1\%) and L2-L3 in 4 (11.8\%) patients. Twelve (35.3\%) patients had one level of stenosis, $11(32.4 \%)$ had two levels, 9 (26.5\%) had three levels and two $(5.9 \%)$ had four levels (Table 1$)$.

Four $(11.8 \%)$ patients received the treatment one time, $20(58.8 \%)$ underwent two interventions, 7 (20.6\%) had three interventions, two $(5.9 \%)$ received four interventions and one (2.9\%) patient underwent five interventions. No significant or fatal adverse effects were reported following the treatment. The average follow-up period was $12.9 \pm 1.1$ months.

After the patients underwent fluoroscopically guided transforaminal epidural dry needling, the mean VAS pain score decreased by $2.7 \pm 3.0$ points (from $7.3 \pm 2.0$ at baseline to $4.6 \pm 2.5$ at follow-up) $(p<0.01)$, the per cent disability score on the ODI decreased by $15.9 \pm 19.5 \%$ 
Table 1 Baseline patient characteristics

\begin{tabular}{|c|c|c|c|c|c|c|c|c|c|}
\hline \multirow[t]{2}{*}{ Patient } & \multirow[t]{2}{*}{ Age (yr) } & \multirow[t]{2}{*}{ Sex } & \multirow[t]{2}{*}{ Pain Duration (months) } & \multirow[t]{2}{*}{ VAS } & \multirow[t]{2}{*}{ ODI (\%) } & \multicolumn{4}{|c|}{ Stenosis level } \\
\hline & & & & & & L2-L3 & L3-L4 & L4-L5 & L5-S1 \\
\hline 1 & 31 & $M$ & 2 & 8 & 82 & & & $\circ$ & \\
\hline 2 & 35 & $\mathrm{~F}$ & 4 & 9 & 66 & & & $\circ$ & \\
\hline 3 & 36 & M & 140 & 8 & 42 & & & $\circ$ & \\
\hline 4 & 54 & $\mathrm{~F}$ & 72 & 10 & 18 & & & $\circ$ & $\circ$ \\
\hline 5 & 55 & $\mathrm{~F}$ & 36 & 7 & 44 & & & $\circ$ & \\
\hline 6 & 56 & $\mathrm{~F}$ & 36 & 10 & 38 & & $\circ$ & $\circ$ & \\
\hline 7 & 57 & M & 420 & 5 & 42 & & & $\circ$ & \\
\hline 8 & 58 & F & 120 & 6 & 32 & & $\circ$ & $\circ$ & \\
\hline 9 & 59 & M & 24 & 5 & 22 & & $\circ$ & $\circ$ & \\
\hline 10 & 59 & $\mathrm{~F}$ & 8 & 5 & 32 & & & $\circ$ & \\
\hline 11 & 60 & $F$ & 108 & 6 & 8 & $\circ$ & $\circ$ & & $\circ$ \\
\hline 12 & 60 & $\mathrm{~F}$ & 2 & 9 & 40 & & $\circ$ & $\circ$ & $\circ$ \\
\hline 13 & 62 & $F$ & 36 & 5 & 22 & & & $\circ$ & \\
\hline 14 & 63 & $F$ & 48 & 6 & 20 & & $\circ$ & ○ & \\
\hline 15 & 64 & $M$ & 18 & 5 & 52 & & & $\circ$ & $\circ$ \\
\hline 16 & 64 & $M$ & 36 & 6 & 36 & & $\circ$ & $\circ$ & \\
\hline 17 & 65 & $F$ & 18 & 10 & 50 & & & $\circ$ & \\
\hline 18 & 65 & $F$ & 240 & 6 & 28 & & $\circ$ & $\circ$ & $\circ$ \\
\hline 19 & 65 & $F$ & 36 & 5 & 27 & & $\circ$ & $\circ$ & \\
\hline 20 & 66 & $F$ & 4 & 5 & 36 & & $\circ$ & & $\circ$ \\
\hline 21 & 66 & $F$ & 6 & 8 & 62 & & $\circ$ & & $\circ$ \\
\hline 22 & 68 & $\mathrm{M}$ & 96 & 5 & 44 & $\circ$ & $\circ$ & $\circ$ & $\circ$ \\
\hline 23 & 68 & $M$ & 5 & 10 & 66 & & ○ & ○ & $\circ$ \\
\hline 24 & 69 & F & 60 & 8 & 30 & & $\circ$ & ○ & $\circ$ \\
\hline 25 & 70 & $F$ & 120 & 9 & 48 & & $\circ$ & ○ & $\circ$ \\
\hline 26 & 70 & $F$ & 92 & 10 & 54 & & & ○ & $\circ$ \\
\hline 27 & 71 & F & 80 & 10 & 40 & & $\circ$ & $\circ$ & $\circ$ \\
\hline 28 & 72 & $M$ & 36 & 5 & 36 & $\circ$ & $\circ$ & $\circ$ & \\
\hline 29 & 72 & $F$ & 96 & 6 & 22 & & & $\circ$ & \\
\hline 30 & 73 & $F$ & 36 & 8 & 30 & $\circ$ & $\circ$ & $\circ$ & $\circ$ \\
\hline 31 & 76 & $F$ & 120 & 10 & 60 & & & $\circ$ & \\
\hline 32 & 76 & $\mathrm{~F}$ & 80 & 8 & 68 & & & ○ & \\
\hline 33 & 77 & $F$ & 60 & 6 & 40 & & & $\circ$ & \\
\hline 34 & 77 & $\mathrm{~F}$ & 24 & 10 & 70 & & $\circ$ & $\circ$ & ○ \\
\hline
\end{tabular}

VAS, visual analogue scale pain score; ODI, the Oswestry Disability Index score.

(from $41.4 \pm 17.2 \%$ at baseline to $25.5 \pm 12.6 \%$ at followup) $(p<0.01)$, and the average of the self-rated improvement following the intervention was $52.6 \pm 33.1 \%$ $(p<0.01)$. The VAS scores indicated that $14(41.2 \%)$ patients reported a "good" to "excellent" treatment response, $11(32.4 \%)$ patients had a "good" to "excellent" treatment response on the ODI and $22(64.7 \%)$ patients had a "good" to "excellent" treatment response on the self-rated improvement scale (Table 2).

\section{Discussion}

The VAS and ODI scores improved significantly following fluoroscopically guided transforaminal epidural dry needling, and nearly $65 \%$ of the patients rated their response as "good" to "excellent" on the self-rated improvement assessment. These results suggest that fluoroscopically guided transforaminal epidural dry needling is an effective intervention for managing LSS.

The technique was developed from our clinical experience in dry needling of neural tissues. We have found that the technique has a beneficial effect on various chronic pain conditions including spinal pain, without the addition of medication $[19,20]$. For the treatment mechanisms of our intervention, we hypothesise that dry needling improves nerve mobility, reduces neuronal hypersensitivity and promotes the natural healing process, resulting in improvement of painful conditions. 
Table 2 Treatment responses to fluoroscopically guided transforaminal epidural dry needling

\begin{tabular}{|c|c|c|c|c|c|c|c|}
\hline \multirow[t]{2}{*}{ Outcome measure } & \multicolumn{3}{|c|}{ Average change } & \multicolumn{4}{|c|}{ Percent change } \\
\hline & Baseline & Follow-up & $P$ & $\begin{array}{r}\text { Excellent } \\
(\geq 75 \%) \\
\end{array}$ & $\begin{array}{c}\text { Good } \\
(50-74 \%)\end{array}$ & $\begin{array}{c}\text { Fair } \\
(25-49 \%) \\
\end{array}$ & $\begin{array}{c}\text { Poor } \\
(<25 \%)\end{array}$ \\
\hline VAS pain score (points) & $7.3 \pm 2.0$ & $4.6 \pm 2.5$ & $<0.0001$ & $7(20.6)$ & $7(20.6)$ & $4(11.8)$ & $16(47.1)$ \\
\hline Percent disability of ODI score (\%) & $41.4 \pm 17.2$ & $25.5 \pm 12.6$ & $<0.0001$ & $7(20.6)$ & $4(11.8)$ & $6(17.6)$ & $17(50.0)$ \\
\hline Self-rated improvement following treatment (\%) & - & $52.6 \pm 33.1$ & $<0.0001$ & $10(29.4)$ & $12(35.3)$ & $4(11.8)$ & $8(23.5)$ \\
\hline
\end{tabular}

VAS, visual analogue scale pain score; ODI, Oswestry Disability Index score.

Although we could not demonstrate the presence of adhesion between the nerve root and surrounding tissue, surgeons report that adhesion is a common pathological condition in LSS and studies demonstrate that it plays an important role in the pathogenesis of lumbar spinal disorders, including LSS [21]. We believe that dry needling into the intervertebral foramen using a $1.2-\mathrm{mm}$ diameter needle is sufficient to release the adhesion between the nerve root and surrounding tissue and thereby decompress the lumbar spinal nerve, thus improving nerve mobility and alleviating pain.

Most of the patients who were not sedated and locally anaesthetised with lidocaine reported that they experienced a sensation like an electric current running through their back or lower extremity when the needle was advanced into their epidural space. We could observe twitch responses of the muscles innervated by the affected spinal nerves in the subjects during the procedure. Based on the patients' experiences and our observation, we hypothesised that needling induces neuronal reflex, thereby reducing hypersensitivity of the neural tissue.

Lumbar spinal stenosis causes prolonged blocking of the cauda equina and nerve root(s). Studies have demonstrated that hindering neuronal impulses for a period of time causes hypersensitivity of the denervated organs $[22,23]$. Dry needling produces minute wounds. The wounds generate a current of injury continuously for several days or weeks, which promotes the natural healing process and a decline in denervation hypersensitivity $[24,25]$. Furthermore, blood flow increases around the injured area, which may relieve localised ischaemia or vasoconstriction.

A transforaminal epidural contrast injection test using a spinal needle was conducted to examine whether the flexed Round Needle effectively reached the posterior epidural space, the primary target of our intervention. When the spinal needle was advanced transforaminally in its original straight form, we found it difficult to contact the canal side of the inferior articular process between the facet joint and pedicle and advance further into the anterior epidural space. However, when the tip was curved (Figure 4A), one could easily contact the canal side of the inferior articular process between the facet joint and pedicle and reach the posterior epidural space (Figures $4 \mathrm{~B}$ and $4 \mathrm{C}$ ), indicating that the bent needle allowed us to reach the primary target of our intervention.

While performing interventions, we were careful to avoid complications such as haematoma, dural puncture and anterior spinal artery syndrome, and no evidence of complications was detected in, or reported by, the patients. We applied the intervention clinically after confirming its safety in cadaveric examinations. Figure 5 shows a cadaveric study performed to evaluate the safety of fluoroscopically guided transforaminal epidural dry needling. The flexed Round Needle was inserted transforaminally into the right L3-L4 intervertebral foramen of a cadaver under $\mathrm{C}$-arm fluoroscopic guidance. An endoscope was introduced following the same route and traced the needle (Figures 5A, B and 5C). The study demonstrated that our intervention does not injure critical spinal structures, including the spinal nerve, dura mater and thecal sac. In clinical practice, the needle primarily approaches the posterior epidural space. However, demonstrating the approach to the posterior epidural space in this cadaveric study was impossible because the epidural space was congested with the needle and endoscope, and limited space was available for J-turning the endoscope to visualise the posterior epidural space.

We believe that the streamlined shape, solid but flexible body and round blunt tip of the specially designed needle minimise tissue damage. In most cases, the needle is advanced to the outer-third of the pedicle and moved along the canal side of the inferior articular process between the facet joint and pedicle. The needle is introduced into the "safe triangle", which was suggested by Bogduk et al. [26] as the roof made up by the pedicle, a tangential base that corresponds to the exiting nerve root, and the lateral border of the vertebral body.

Our technique shares some aspects of conventional spinal interventions for LSS, such as transforaminal epidural steroid injection and epiduroscopic adhesiolysis. However, it has several unique features and advantages. While the conventional transforaminal epidural steroid injection approach is similar to ours, we do not use corticosteroid, which can cause collagen degeneration and 


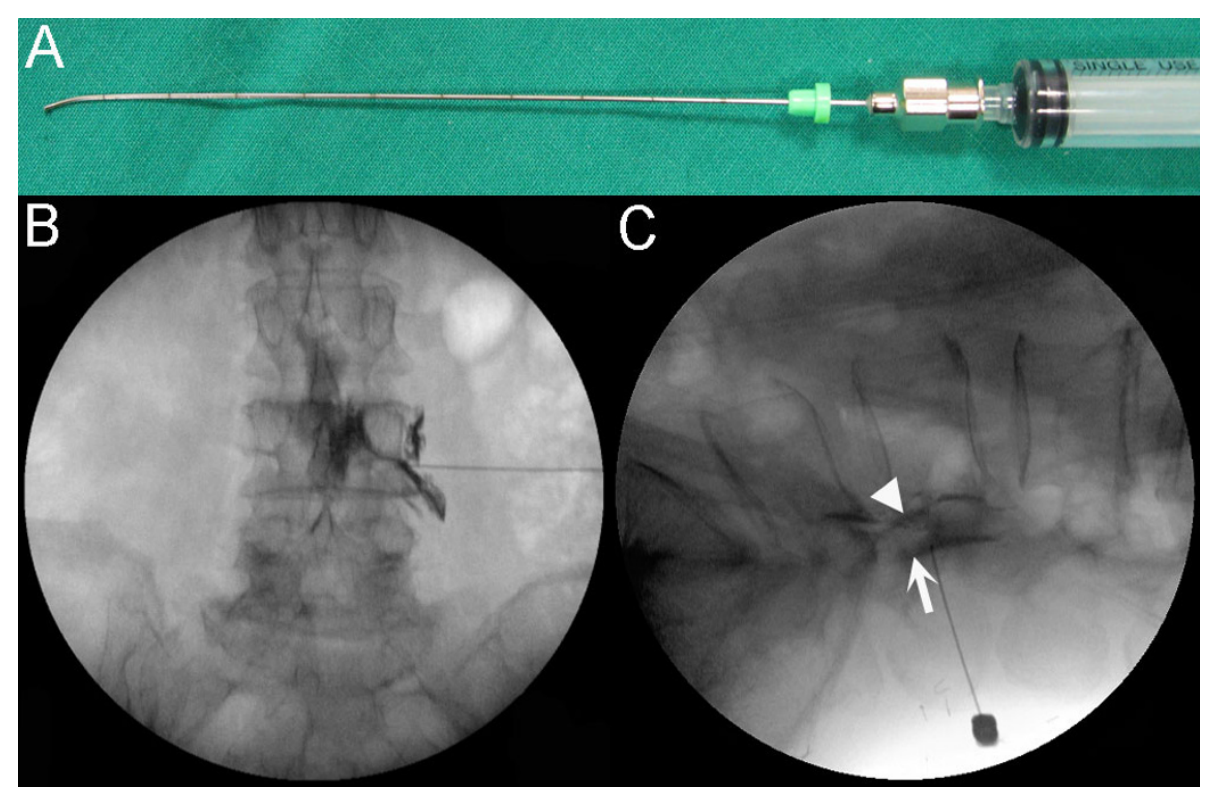

Figure 4 Transforaminal epidural contrast injection test to demonstrate that the specially designed needle effectively reached the target structures. The curved spinal injection needle used in the test (A). The anteroposterior fluoroscopy image shows a needle that has been advanced transforaminally at the L4-L5 level in the same manner as that used in the treatment and contrast agent flowing through the epidural space (B). The lateral fluoroscopy image. The triangle ( $\mathbf{v})$ indicates contrast agent in the anterior epidural space when the needle was used in its original straight form. The arrow $(\uparrow)$ indicates contrast agent in the posterior epidural space when the curved needle was used. The curved needle contacted the canal side of the inferior articular process between the facet joint and pedicle (C).

disturb the healing process, although it may also reduce inflammation. The spread of corticosteroid into critical structures beyond the treatment target is rare, but it can cause serious complications [27]. Our technique uses a needle with a blunt, round tip, so the treatment effect is limited to the needling points. An epiduroscope is introduced into the sacral hiatus and advanced into the interlaminar posterior epidural space. When an adhesion or thick connective tissue is detected in the epidural space, it is broken down using saline injections and steroid or local anaesthetic [28]. The lumbar epidural space in adults is segmented and discontinuous and may impede the passage of the epiduroscope and cause its misplacement [29]. Using a needle in the transforaminal route may break down adhesion between the nerve root and surrounding tissues. It also allows access to the upper lumbar levels.

The design of this study, a case series, poses a limitation, and further studies or randomised clinical trials are needed to evaluate the efficacy of our technique

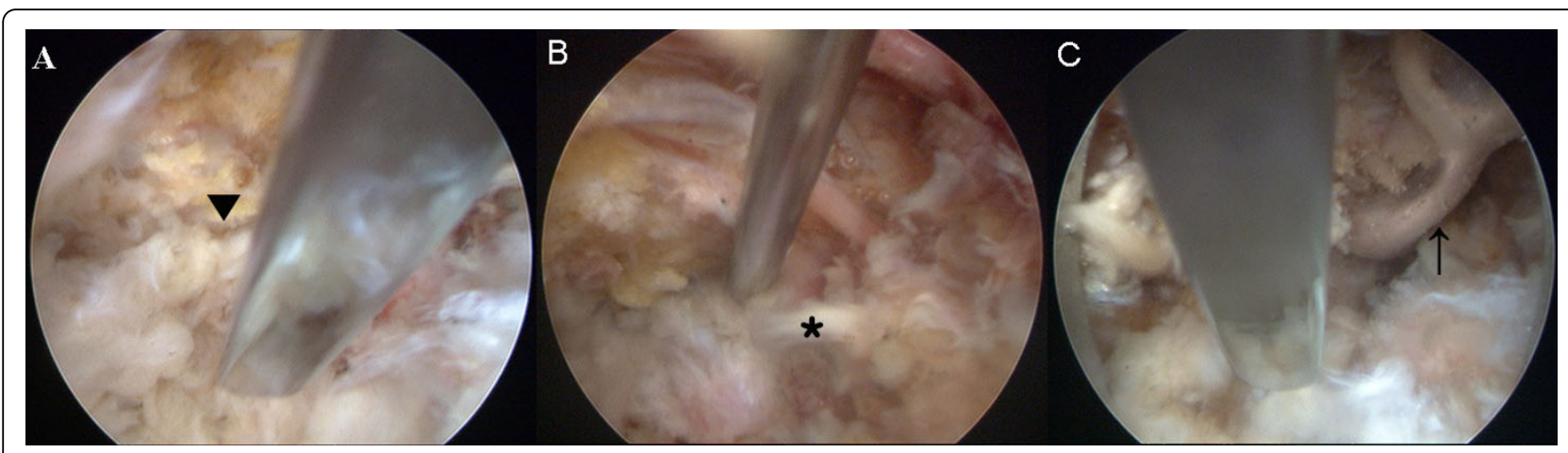

Figure 5 A cadaveric examination used to evaluate the safety of fluoroscopically guided transforaminal epidural dry needling. The flexed Round Needle contacted and passed by the facet joint ( $\mathbf{v})$ is advanced into the intervertebral foramen (A). The needle passes over the posterior longitudinal ligament $\left(^{*}\right)$ and reaches the anterior epidural space in the same manner as with the anterior epidural approach (B). After removing the posterior longitudinal ligament and dura mater by cauterisation, the tip of the needle is found to be located under the thecal sac $(\uparrow)$ without contacting it (C). 
compared with other treatment methods for LSS. We have suggested possible treatment mechanisms, but they are not fully supported by other studies. Further research is needed to evaluate our hypothesis, including animal studies. Although this study suggests that our technique is effective for managing LSS, fewer than half of the subjects reported a "good" to "excellent" treatment response as evaluated by the VAS and ODI scores. Further work is necessary to increase the favourable treatment response.

\section{Conclusions}

We developed a minimally invasive interventional technique using a specially designed needle to treat LSS. Thirty-four patients with LSS underwent fluoroscopically guided transforaminal epidural dry needling. At the level of the stenosis, a specially designed flexed Round Needle was inserted 8-12 cm lateral to the midline and advanced to a position between the anterior side of the facet joint and pedicle. It was advanced medially and backed laterally along the canal side of the inferior articular process between the facet joint and pedicle. The procedure was completed when the resistance at the tip of the needle was markedly reduced. The treatment outcome was assessed using a VAS pain score, the ODI and self-rated improvement. A significant improvement was found in each measure after the procedure. These results suggest that fluoroscopically guided transforaminal epidural dry needling is an effective intervention for managing LSS.

\section{Additional material}

Additional file 1: Windows Media Video. Fluoroscopically guided transforaminal epidural dry needling for lumbar spinal stenosis using a specially designed needle. A movie demonstrating the technique

\section{Acknowledgements}

We thank Dr. Kyeong-Hee Kim for her help with data collection.

\author{
Author details \\ ${ }^{1}$ Chronic Pain Management Centre, Cha Biomedical Centre, Kangnam Cha \\ Hospital, Cha University, 605, Yeoksam-Dong, Kangnam-Ku, Seoul, Republic \\ of Korea. ${ }^{2}$ Centre for Interventional Pain Management, Daejeon Woori \\ Hospital, 1419, Metrozone, Doonsan-Dong, Seo-Ku, Daejeon, 302-531, \\ Republic of Korea.
}

\section{Authors' contributions}

KA developed the fluoroscopically guided transforaminal epidural dry needling technique and the specially designed needle used in this study. $\mathrm{HJJ}$ analysed the data and wrote the manuscript. TKL and YSL collected the data. All authors read and approved the final manuscript.

\section{Competing interests}

KA developed the 'Round Needle', the specially designed needle used in this study. He possesses Korean patent 10-2004-41689 on the needle. However, he has no stocks or ownership in the company manufacturing the needle and has not been compensated for possessing the patent by any individual or organisation. None of the authors receives reimbursements, fees, funding or salary from any individual or organisation related to the content of the manuscript.

Received: 26 February 2010 Accepted: 11 August 2010 Published: 11 August 2010

\section{References}

1. Fritz JM, Delitto A, Welch WC, Erhard RE: Lumbar spinal stenosis: a review of current concepts in evaluation, management, and outcome measurements. Arch Phys Med Rehabil 1998, 79:700-708.

2. Weinstein PR: Diagnosis and management of lumbar spinal stenosis. Clin Neurosurg 1983, 30:677-697.

3. Binder DK, Schmidt MH, Weinstein PR: Lumbar spinal stenosis. Semin Neurol 2002, 22:157-166.

4. Katz JN, Harris MB: Clinical practice. Lumbar spinal stenosis. N Engl J Med 2008, 358:818-825

5. Elsig JP, Kaech DL: Imaging-based planning for spine surgery. Minim Invasive Ther Allied Technol 2006, 15:260-266.

6. Gunn CC: Radiculopathic pain: Diagnosis and. treatment of segmental irritation or sensitization. J Musculoske Pain 1997, 5:119-134.

7. Jenis $L G, A n H S$, Gordin R: Foraminal stenosis of the lumbar spine: a review of 65 surgical cases. Am J Orthop 2001, 30:205-211.

8. Jarvik JJ, Hollingworth W, Heagerty P, Haynor DR, Deyo RA: The Longitudinal Assessment of Imaging and Disability of the Back (LAIDBack) Study: baseline data. Spine 2001, 26:1158-1166.

9. Borenstein DG, O'Mara JW Jr, Boden SD, Lauerman WC, Jacobson A, Platenberg C, Schellinger D, Wiesel SW: The value of magnetic resonance imaging of the lumbar spine to predict low-back pain in asymptomatic subjects: a seven-year follow-up study. J Bone Joint Surg Am 2001, 83A:1306-1311.

10. Bogduk $\mathrm{N}$ : Clinical anatomy of the lumbar spine and sacrum. Edinburgh: Churchill Livingstone, 42005.

11. Porter RW: Spinal stenosis and neurogenic claudication. Spine 1996, 21:2046-2052.

12. Okuda T, Fujimoto Y, Tanaka N, Ishida O, Baba I, Ochi M: Morphological changes of the ligamentum flavum as a cause of nerve root compression. Eur Spine J 2005, 14:277-286.

13. Kobayashi S, Kokubo Y, Uchida K, Yayama T, Takeno K, Negoro K, Nakajima $H$, Baba $H$, Yoshizawa $H$ : Effect of lumbar nerve root compression on primary sensory neurons and their central branches: changes in the nociceptive neuropeptides substance $P$ and somatostatin. Spine 2005, 30:276-282.

14. Ahn K, Lee YJ, Kim EH, Yang SM, Lim TK, Kim YS, Jhun HJ: Interventional microadhesiolysis: a new nonsurgical release technique for adhesive capsulitis of the shoulder. BMC Musculoskelet Disord 2008, 9:12.

15. Yonetake T, Sekiguchi M, Konno S, Kikuchi S, Kanaya F: Compensatory neovascularization after cauda equina compression in rats. Spine 2008, 33:140-145.

16. Fairbank JC, Pynsent PB: The Oswestry Disability Index. Spine 2000 25:2940-2952.

17. Sirvanci M, Bhatia M, Ganiyusufoglu KA, Duran C, Tezer M, Ozturk C, Aydogan M, Hamzaoglu A: Degenerative lumbar spinal stenosis: correlation with Oswestry Disability Index and MR imaging. Eur Spine J 2008, 17:679-685.

18. Jou IM, Chern TC: Sonographically assisted percutaneous release of the a1 pulley: a new surgical technique for treating trigger digit. $J$ Hand Surg 2006, 31:191-199.

19. Ahn K, Lee YJ, Lee SC, Lee CW, Lee YC: Clinical effect of fluoroscopy guided interventional muscle and nerve stimulation (IMNS) on intractable spinal origin pain. Korean J Anesthesiol 2004, 47:96-100.

20. Kim EH: Clinical effects of fluoroscopy guided interventional microadhesiolysis and nerve stimulation (FIMS) on cervical zygapophyseal joints in patients with chronic cervical radicular pain. J Korean Pain Soc 2007, 20:31-39.

21. Miyamoto $H$, Dumas GA, Wyss UP, Ryd L: Three-dimensional analysis of the movement of lumbar spinal nerve roots in nonsimulated and simulated adhesive conditions. Spine 2003, 28:2373-2380.

22. Lorković $\mathrm{H}$ : Supersensitivity to $\mathrm{ACh}$ in muscles after prolonged nerve block. Arch Int Physiol Biochim 1975, 83:771-781. 
23. Antony MT, Tonge DA: Effects of denervation and botulinum toxin on muscle sensitivity to acetylcholine and acceptance of foreign innervation in the frog. J Physiol 1980, 303:23-31.

24. Gunn CC, Milbrandt WE, Little AS, Mason KE: Dry needling of muscle motor points for chronic low-back pain: a randomized clinical trial with long-term follow-up. Spine 1980, 5:279-291.

25. Balakatounis KC, Angoules AG: Low-intensity electrical stimulation in wound healing: Review of the efficacy of externally applied currents resembling the current of injury. Eplasty 2008, 8:e28.

26. Vad VB, Bhat AL, Lutz GE, Cammisa F: Transforaminal epidural steroid injections in lumbosacral radiculopathy: a prospective randomized study. Spine 2002, 27:11-16.

27. Kennedy DJ, Dreyfuss P, Aprill CN, Bogduk N: Paraplegia following imageguided transforaminal lumbar spine epidural steroid injection: two case reports. Pain Med 2009, 10:1389-1394.

28. Igarashi T, Hirabayashi Y, Seo N, Saitoh K, Fukuda H, Suzuki H: Lysis of adhesions and epidural injection of steroid/local anaesthetic during epiduroscopy potentially alleviate low back and leg pain in elderly patients with lumbar spinal stenosis. Br J Anaesth 2004, 93:181-187.

29. Anatomy of the Epidural Space. [http://www.arachnoiditis.info/content/ articles/epidural_space_anatomy/Epidural\%20Space\%20Anatomy.htm].

\section{Pre-publication history}

The pre-publication history for this paper can be accessed here: http://www.biomedcentral.com/1471-2474/11/180/prepub

doi:10.1186/1471-2474-11-180

Cite this article as: Ahn et al.: Fluoroscopically guided transforaminal epidural dry needling for lumbar spinal stenosis using a specially designed needle. BMC Musculoskeletal Disorders 2010 11:180

\section{Submit your next manuscript to BioMed Central and take full advantage of:}

- Convenient online submission

- Thorough peer review

- No space constraints or color figure charges

- Immediate publication on acceptance

- Inclusion in PubMed, CAS, Scopus and Google Scholar

- Research which is freely available for redistribution

Submit your manuscript at www.biomedcentral.com/submit 